\title{
Some Applications of Infrared Thermography for Antenna Characterization in the Polytechnic University of Catalonia
}

\author{
by J. M. González-Arbesú and J. Romeu
}

Universitat Politécnica de Catalunya (UPC), Barcelona, Spain

\begin{abstract}
Infrared Thermography has been applied with success to antenna diagnostic and even to power pattern determination of antennas. Some of the experiences carried out at UPC in relation with this technique are presented.
\end{abstract}

\section{Introduction}

The radiation power pattern determination of antennas is typically carried out using near-field techniques in anechoic chambers. It requires the measurement of the electric field using a reference antenna (probe) placed in the near-field of the AUT. Field samples are acquired on a surface surrounding the antenna under test (AUT) and a near to far field transformation is done to compute the far field pattern of the antenna. However, electromagnetic field measurement in the close vicinity of radiant objects is expensive, time consuming and it is severely affected by the interaction between the antenna and the probe.

To overcome these limitations, and following the outstanding works of Prof. Norgard [1,2] we have used InfraRed Thermography (IRT) as a mean to measure electromagnetic near-fields for fast and non-invasive radiation pattern determination of antennas. This technique uses an IR camera to measure the radiation in the IR band emitted by a thin film of lossy material when heated by the electromagnetic waves radiated by the AUT.

\section{Radiofrequency power and temperature rise above ambient}

Electromagnetic waves impinging on lossy materials partially transform their energy into heat. As a result the material raises its temperature above the environment. The RF power density $P_{a b s}$ absorbed by a planar screen ( $t$ : thickness) of lossy material is related to conductivity $\sigma$, to the complex parts of permittivity $\varepsilon$ " and permeability $\mu$ ", and to radian frequency $\omega$ by Eq. (1)

$$
P_{a b s}=\int_{0}^{\mathrm{t}}\left[\sigma|\vec{E}|^{2}+\omega \varepsilon^{\prime \prime}|\vec{E}|^{2}+\omega \mu^{\prime \prime}|\vec{H}|^{2}\right] d z
$$

Accordingly, a screen sensitive to the electric or magnetic field can be configured with a proper choice of the electromagnetic properties of the material $(\sigma$, $\varepsilon "$, and $\left.\mu^{\prime \prime}\right)$. As a function of the incident field on the detection screen $\boldsymbol{E}_{\text {inc }}$, the power density $P_{a b s}$ absorbed by a lossy material sensitive to the electric field radiated by a source is,

$$
P_{a b s}=\Gamma_{a b s}\left|\vec{E}_{i n c}\right|^{2}
$$

where $\Gamma_{a b s}$ is the absorption coefficient. $\Gamma_{a b s}$ is computed using the duality between plane waves on infinite dielectrics and transmission lines from the dimensions and electromagnetic $(\sigma, \varepsilon$, and $\mu$ ) properties of the lossy material. The absorption 


\section{http://dx.doi.org/10.21611/qirt.2004.053}

coefficient can be measured using a vector network analyser through reflection and transmission measurements.

The power absorbed by the screen is transformed into heat, raising its temperature $T$ from ambient $T_{a m b}$. For a lossy material attached to a thermal insulator, the non-linear equation that relates absorbed power density and temperature on the detection screen is,

$$
P_{a b s}=h\left(T-T_{a m b}\right)^{p}+\varepsilon_{I R} \sigma_{S B}\left(T^{4}-T_{a m b}^{4}\right)
$$

being $\varepsilon_{I R}$ the infrared emissivity of the material, $\sigma_{S B}$ the Stefan-Boltzman constant, and $h$ and $p$ empirical ([3]) figures that depend on the dimensions and orientation of the screen. From experimental measurements of figure 1 (for a small detection screen of $3 \mathrm{~cm} \times 1 \mathrm{~cm}$ ), and due to the inaccuracy in the determination of the thermal coefficient $h$, we suggest a linear approximation between temperature rise $\left(T-T_{a m b}\right)$ and absorbed power density useful in practical applications where absolute measurements are not needed (such as for radiation pattern determination). It is a very simple approach for solving Eq. (3) without knowing accurately the physical properties of the material. Hence,

$$
\left|\vec{E}_{\text {inc }}\right|^{2} \approx K \Delta T=K\left(T-T_{a m b}\right)
$$

being $K$ a constant needed for absolute measurements of electric field intensities (and consequently requiring a calibration).

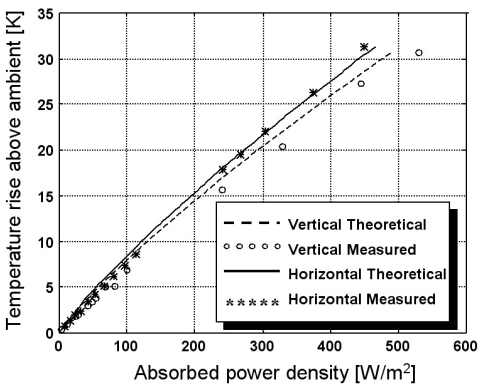

Fig. 1. Theoretical and measured temperature rise above ambient versus absorbed power density for horizontally and vertically oriented detection screens.

The linearization of Eq. (3) is also helpful for solving the heat equation that rules the temperature rise of a detection screen [4] for a given $P_{a b s}$. In fact, temperature rise $\Delta T(\boldsymbol{r})$ can be related to the absorbed power density $P_{a b s}(\mathbf{r})$ by a bidimensional convolution through the hot-spot response $h(r)$ of the screen $[4,5]$

$$
\Delta T(\vec{r})=h(\vec{r}) * * P_{a b s}(\vec{r})
$$

From the thermal map of the screen it is possible to retrieve $P_{a b s}$ by solving the inverse problem of Eq. (5).

\section{Measurement set-up}

IR thermography measurements require four elements: a detection screen sensitive to electric fields, an IR camera to measure the thermal distributions on the detection screen, a RF source (preferably a solid state amplifier) to feed the antennas with enough power to raise the temperature of the detection screen (less than $50 \mathrm{~W}$ is usually enough), and a personal computer to control the measurement 
procedure and to carry out the data processing. We have fabricated a detection screen using a carbon paper (conductivity: $7.6 \mathrm{~S} / \mathrm{m}$, emissivity: 0.9, thickness: $80 \mu \mathrm{m}$ ) attached to a thermal insulator (stirofoam) that reflects a $2 \%$ of the incoming power and absorbs almost a $22 \%$. The IR camera operates in the medium IR $(3.6-5 \mu \mathrm{m})$ and its thermal sensitivity is $0.1^{\circ} \mathrm{C}$ at $30^{\circ} \mathrm{C}$ (without averaging) with a dynamic range of 12 bits. To reduce the noise in the measurement of temperatures the averaging of images is mandatory. The variance of the noise in each pixel (temporal noise with a Gaussian density function) is then reduced by a factor of $N$, being $N$ the number of images averaged. The camera detector is a 240 x 320 pixel PtSi FPA cooled by a rotary Stirling microcooler. The thermal map of the whole detection screen is captured in a snapshot and transferred to the computer for a further post-processing.

Measurements are carried out inside an anechoic chamber to avoid the exposure of the human operator to high electric field levels. The detection screen was horizontally located to avoid the blurring of the thermal images due to thermal convection. However, in some experiments (mainly when temperatures were not very high) the detection screen was vertically oriented and the data processed to compensate this effect.

\section{Electromagnetic Field Intensity Measurements}

To assess the validity of the technique for the measurement of radiation patterns of antennas several experiments involving electric field intensity measurements with IR thermograms were carried out. Successful results $[5,6]$ were found for the near fields generated for wire antennas, and also for the self-similar behaviour of multiband prefractal antennas. This technique also revealed better resolution and important time savings than standard techniques of current reconstruction from near-field/far-field measurements when applied to aperture antennas. Figure 2 shows the normalized field intensities of an eight-element slotted waveguide array retrieved from a standard spherical near-field facility versus the normalized field intensities measured with the infrared technique. Better resolution (much lower than $N / 2$ ) of the IR technique is clearly shown from the figure.

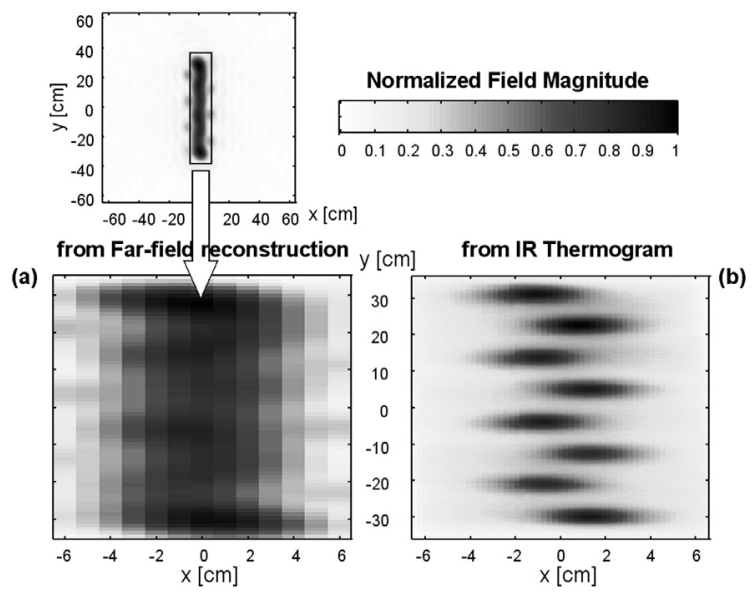

Fig. 2. Normalized electrical field magnitude of an 8-element slotted waveguide array retrieved from far-field measurements (a) and measured with an IR thermogram (b). 


\section{Radiation pattern determination using Infrared Thermography}

A Cartesian near-field measurement set-up has been used for the radiation pattern determination of antennas with narrow beams. Electric field samples are acquired in the near-field in a planar surface in front of the antenna, and by means of a near to far-field transformation the radiation pattern of the AUT is computed. IR thermography is used for an indirect and quick electric field intensity measurement. If the aperture size of the AUT is known, with just one measurement plane is enough to retrieve the phase distribution of the field using a phase retrieval algorithm. Otherwise, two or more planes are needed. The current reconstruction algorithm [7] was preferred among others [4] with larger execution times and after deceiving results. The current reconstruction algorithm is the one that provided better results.

Two aperture antennas were characterized using this technique, a pyramidal horn and an eight-element slotted waveguide array. The radiation pattern for the pyramidal horn was computed using only one measurement plane at $0.300 \mathrm{~m}(2.45 \lambda)$ from the aperture, whereas for the waveguide array we used two measurement planes at $0.295 \mathrm{~m}(2.41 \lambda)$ and $0.465 \mathrm{~m}(3.80 \lambda)$. The plane sizes were, respectively, $1.840 \mathrm{~m} \times 1.000 \mathrm{~m}$ and $1.600 \mathrm{~m} \times 0.900 \mathrm{~m}$ for both antennas. Thermal images were downsampled to accomplish the sampling criteria and the phase retrieval algorithm was used with the initial knowledge of the aperture size. The solutions settled before than 50 iterations, although we carried on the iterative algorithms till 1000 iterations to assess the validity of the solution. Finally, the retrieved complex field was zeropadded $(128 \times 128)$ to increase the resolution of the plane wave spectrum and, consequently, the resolution of the radiation patterns. Figure 3 shows the main cuts of the radiation patterns of the pyramidal horn and the slotted waveguide array compared with the measured ones in a standard spherical near-field facility.
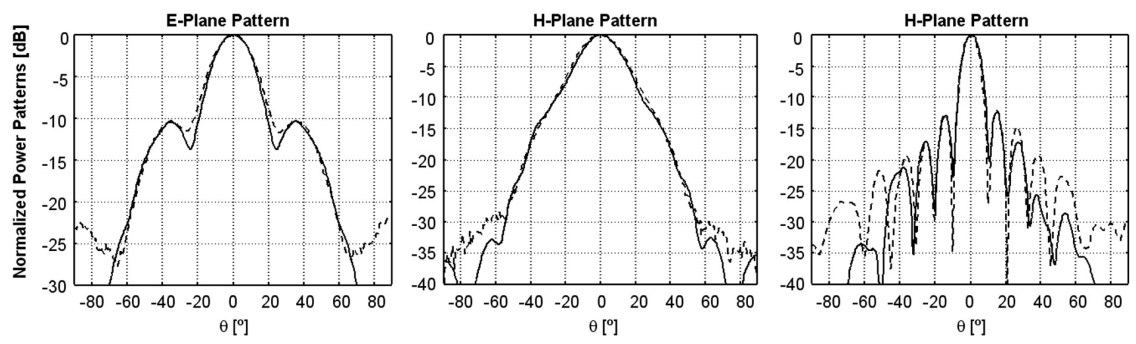

Fig. 3. E and H-plane (left, center) cuts of the radiation pattern of a pyramidal horn. $\mathrm{H}$-plane (right) of the radiation pattern of the 8-element slotted waveguide array.

Using the current reconstruction algorithm for phase retrieval not only the phase distribution in the aperture plane is known but also the magnetic currents on the aperture plane. Figure 4 shows the normalized magnetic currents on the aperture plane for the slotted waveguide array, and the same currents when the fourth aperture of the array is blocked. A scaled schematic of the slotted array is placed near the currents to proof how infrared thermography identifies the blocked aperture.

In spite of these encouraging results the technique potential is constrained by its inability to discriminate polarizations. The detection screen raises its temperature with the absorbed electromagnetic power density radiated by the AUT without accounting for the polarization of the impinging fields. A kind of polarization filtering that does not increase the reflection coefficient of the screen is needed to improve the results and widen the range of antennas where the technique can be applied. In this sense, a small detection screen made with half-wavelength (at $2.45 \mathrm{GHz}$ ) 
metallic dipoles loaded with surface mounted resistors has been tested. The dipoles are distributed on the screen to fulfil the sampling criteria. The temperature of the SMD resistors is the parameter measured with the IR camera because of its relation to the incident field on the screen. First measurements are promising, no cross polarization was measured when the detection screen is placed in front of an open waveguide radiating $10 \mathrm{~W}$ of RF power. However, the non-uniform heating of the screen (also observed in simulations) reveals the need for a calibration prior to any measurement.

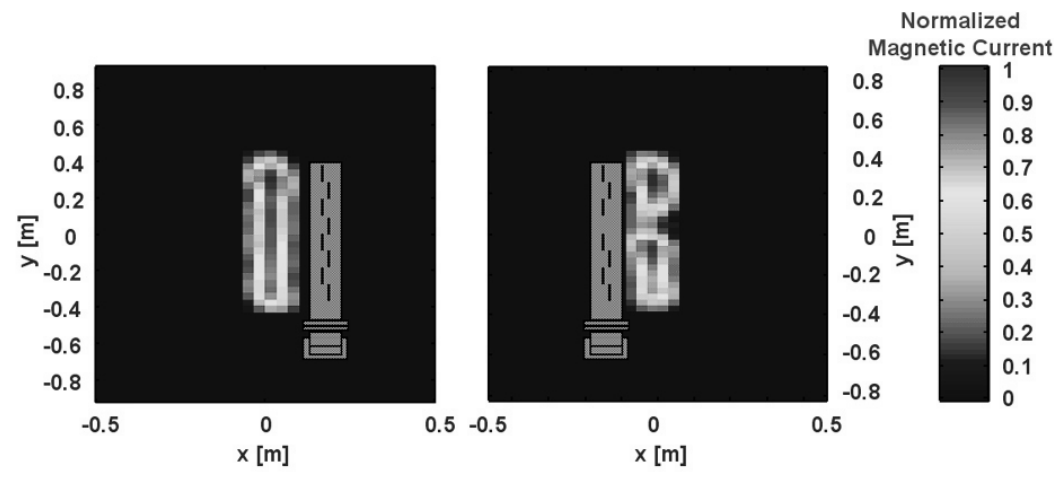

Fig. 4. Normalized magnetic current reconstruction for an 8-element slotted waveguide array (left) and the same array with a blocked element (right).

\section{Error budget of absorbed power density and electric field intensity measured with infrared thermography}

Several are the sources of error when measuring electric field magnitudes [4]. However, for low temperatures raising above ambient $(<10 \mathrm{~K})$ the error source mainly comes from the inaccuracy of the IR camera, but for high temperatures the inaccuracy mainly comes from the difficulty of an accurate knowledge of the convection coefficient $h$. Using infrared thermograms the maximum relative error attainable in the measurement of the absorbed power density is shown in figure 5 (left), for a maximum error of 0.01 in the knowledge of the material emissivity (0.9) and a $0.15 \mathrm{~K}$ error in the knowledge of temperatures. A maximum relative error of $20 \%$ is considered for the convection coefficient [3, pp. 23-24].

For high temperature increments this maximum relative error in $P_{a b s}$ (around a $10 \%$ ) is transferred to a $5 \%$ maximum relative error in the measurement of the electric field magnitude. This accounts for the errors in the measurement of the $S$ parameters when the detection screen is characterized by means of reflection and transmission coefficients in waveguides. From the HP 8753C User's Manual, the uncertainty for the $\left|S_{11}\right|$ parameter is $3.5 \%$ and for $\left|S_{21}\right|$ parameter is about $1.3 \%$ from the true values. Figure 5 (right) shows these maximum relative errors in the measurement of the incident electric field magnitude using IR thermography.

\section{Conclusions}

IR Thermography has revealed a very useful and accurate tool for the diagnostic and fast determination of radiation power patterns of antennas. Even using a simple linear approximation of the non-linear relation between temperature and absorbed power density gives accurate results. IR Thermography deserves 
further research to overcome the polarization discrimination constrain and for a deeper study on the error budget of the technique.

\section{Acknowledgements}

This work was supported by the Spanish "Comisión Interministerial de Ciencia y Tecnología (CICYT)" through the "Ramón y Cajal" Programme, and the grant TIC2003-09317-C03-03.
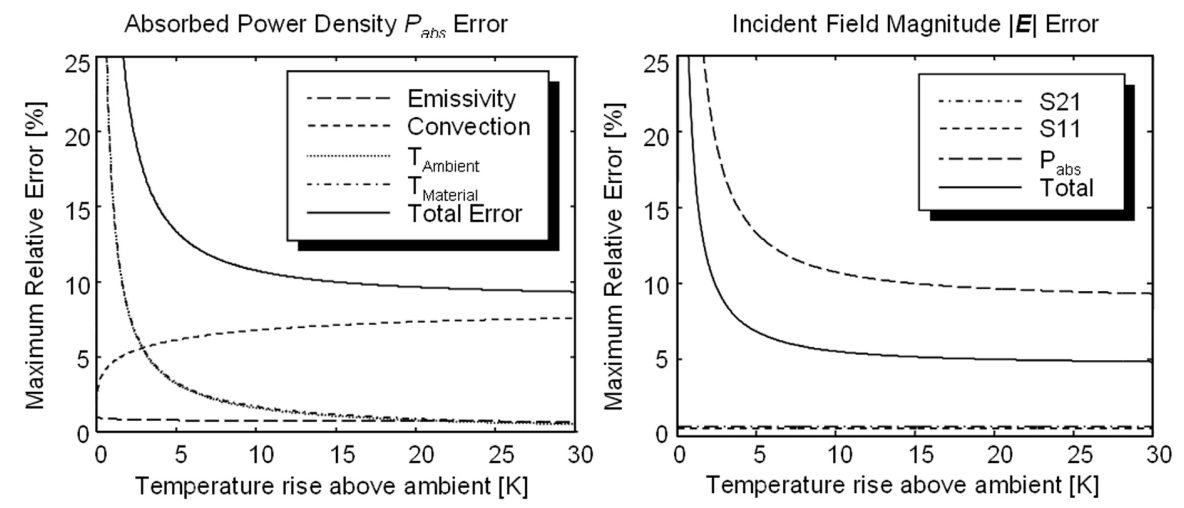

Fig. 5. Maximum relative errors in the measurement of absorbed power density and incident electric field magnitude.

\section{REFERENCES}

[1] J. D. Norgard. Infrared/Microwave Correlation Measurements, Optical Engineering, 33, (1), pp. 85-96, (1994).

[2] J. D. Norgard. Electromagnetic Magnitude \& Phase Measurements from Infrared Thermograms, IEEE Proc. of Aerospace Conference, 2, pp. 145-157, (1997)

[3] F. Incropera and D. Witt. Fundamentals of Heat and Mass Transfer, Singapore: John Wiley \& Sons, $3^{\text {rd }}$ edition, 1990.

[4] José M. González Arbesú. Caracterización de antenas mediante termografías de infrarrojos, PhD disertation (in spanish), UPC, Feb. 2000

[5] J. M. González, A. Aguasca, J. Romeu. Infrared Thermograms Applied to NearField Testing, IEE Electronics Letters, 35, (11), pp. 885-886, (1999).

[6] J. M. González, M. Navarro, C. Puente, J. Romeu, A. Aguasca. Active Zone Self-Similarity of the Fractal-Sierpinski Antenna Verified with Infrared Thermograms, IEE Electronics Letters, 35, (17), pp. 1393-1394, (1999).

[7] R. G. Yaccarino, Y. Rahmat-Samii. Phaseless Bi-Polar Near-Field Measurements and Diagnostics of Array Antennas, IEEE Trans. on Antennas and Propagation, 47,(3), pp. 574-583, (1999). 